\title{
Étude des propriétés élastiques de multicouches métalliques par une méthode d'acoustique picoseconde
}

\author{
E. Romatet, B. Bonello, R. Gohier, J.C. Jeannet et B. Perrin \\ Laboratoire de Dynamique de la Matière Condensée, Université Pierre et Marie Curie, Boîte 136, 4 place \\ Jussieu, 75252 Paris cedex 05, France
}

\begin{abstract}
Résumé : Nous présentons une étude de l'ćlasticité de multicouches métalliques $(\mathrm{Cu} / \mathrm{W}, \mathrm{Ag} / \mathrm{Ni}, \mathrm{Ni} / \mathrm{Mo}) \mathrm{dont}$ l'épaisseur totale n'excède pas $100 \mathrm{~nm}$. La période de composition est comprise entre 3,2 et $20 \mathrm{~nm}$. Les expériences ont été réalisées à l'aide de la "technique ultrasonore picoseconde". Cette technique nous a permis de mesurer la vitesse du son effective des modes longitudinaux de volume se propageant à l'intérieur des hétérostructures et de mettre en évidence sa dépendance vis à vis de la période de composition. Pour les périodes les plus petites, nous observons une décroissance de la vitesse ultrasonore correspondant à un ramollissement lié aux effets interfaciaux. Nous avons également détecté des vibrations haute fréquence s'amortissant sur quelques ps. Ces modes dont la fréquence peut atteindre $810 \mathrm{GHz}$ pourraicnt être attribućs à des modes de surface localisés au centre du second gap de la "mini-zone" de Brillouin du système.
\end{abstract}

\section{INTRODUCTION}

Les techniques les mieux adaptées pour l'exploration des propriétés élastiques des matériaux reposent sur la propagation d'ondes ultrasonores. Pour de telles études, la longueur d'onde acoustique doit être du même ordre de grandeur qu'une longueur caractéristique de la structure étudiée, ce qui pose un problème particulier lorsqu'il s'agit de couches minces ou de multicouches. Dans ce dernier cas, cette longueur caractéristique est la superpériode qui, pour certaines applications, peut être aussi petite que quelques $\mathrm{nm}$. Ceci suppose la mise en œuvre d'ondes ultrasonores dont la fréquence doit être de plusieurs dizaines de $\mathrm{GHz}$, voire d'une centaine de $\mathrm{GHz}$, ce qui rend impossible l'utilisation des techniques conventionnelles. En effet, ces techniques reposent sur l'utilisation de transducteurs piezoélectriques, ce qui restreint le domaine des fréquences accessibles à quelques $\mathrm{GHz}$. Compte tenu de ces limites et d'une vitesse de propagation dans les solides de quelques milliers de $\mathrm{m} / \mathrm{s}$, la longueur d'onde est toujours supérieure à quelques $\mu \mathrm{m}$, ce qui constitue l'épaisseur minimale des échantillons.

Une des techniques les plus utilisées pour l'étude des couches minces reste la diffusion Brillouin de surface qui, selon la géométrie utilisée, concerne des modes de vibration pouvant avoir des fréquences de plusieurs dizaines de $\mathrm{GHz}$. Elle a permis d'obtenir de nombreux résultats intéressants [1], mais elle est mal adaptée à l'étude des propriétés acoustiques de volume des couches minces.

La technique "d'Acoustique Picoseconde" introduite il y a une dizaine d'années par Thomsen et al. [2] permet de s'affranchir de ces différentes limitations. Elle consiste à créer à la surface de l'échantillon des impulsions acoustiques ayant un spectre de fréquences s'étendant sur plusieurs centaines de GHz à l'aide d'impulsions laser ultra-brèves.

Après une rapide description du dispositif expérimental, nous présentons dans cet article quelques résultats expérimentaux obtenus sur différentes multicouches métalliques. Cette technique nous a permis de mesurer les vitesses ultrasonores effectives des modes de volume longitudinaux dans les hétérostructures $\mathrm{Cu} / \mathrm{W}$, $\mathrm{Ni} / \mathrm{Mo}$ et $\mathrm{Ag} / \mathrm{Ni}$ et de mettre en évidence leur dépendance vis à vis de la période du superréseau. En outre, nous détectons pour certaines de ces structures des modes de vibrations localisés à la surface de l'échantillon et dont la fréquence s'étend jusqu'à $810 \mathrm{GHz}(\mathrm{Cu} / \mathrm{W})$. Elles pourraient être attribuées à des modes localisés au centre du second gap de la minj-zone de Brillouin du superréseau. 


\section{DISPOSITIF EXPERIMENTAL}

L'acoustique picoseconde est une technique pompe-sonde dont le principe est le suivant : la surface de l'échantillon est éclairée par une impulsion laser extrêmement brève qui provoque une élévation de température localisée au point d'impact. On montre que la contrainte thermoélastique qui en résulte relaxe dans l'échantillon en excitant certains de ses modes de vibration longitudinaux. Les modes de volume forment une impulsion élastique qui se propage dans l'échantillon, se réfléchit successivement sur l'interface échantillon/substrat puis sur la surface libre engendrant ainsi une suite d'échos qui modifient les propriétés optiques de la surface libre lorsqu'ils y arrivent. On enregistre le changement de réflectivité de la surface du matériau en mesurant l'intensité réfléchie d'une seconde impulsion lumineuse (faisceau sonde). Un chemin optique variable permet de retarder le faisceau sonde. La vitesse du son est déduite du temps séparant deux échos successifs. La résolution temporelle dépend uniquement du déplacement minimum que l'on peut imposer au chemin optique de l'impulsion sonde. Dans le cas de notre montage, elle est définie par la durée de l'impulsion.

\section{DESCRIPTION DES ECHANTILLONS}

Nous avons étudié une série de multicouches métalliques : $\mathrm{Cu} / \mathrm{W}, \mathrm{Ni} / \mathrm{Mo}$ et $\mathrm{Ag} / \mathrm{Ni}$. Les échantillons ont été élaborés et caractérisés par un groupe du Laboratoire de Métallurgie Physique de l'Université de Poitiers [3]. Ils ont été préparés par pulvérisation directe sur un substrat (100) de silicium $(\mathrm{Cu} / \mathrm{W}, \mathrm{Ni} / \mathrm{Mo})$ ou sur un substrat de verre $(\mathrm{Ag} / \mathrm{Ni})$. La période d est comprise entre 5 et $20 \mathrm{~nm}(\mathrm{Cu} / \mathrm{W})$ et entre 3,2 et $7,1 \mathrm{~nm}$ $(\mathrm{Ni} / \mathrm{Mo})$; elle est de 7,5 nm pour l'échantillon $\mathrm{Ag} / \mathrm{Ni}$. Le nombre de bicouches est tel que l'épaisseur totale de chaque échantillon est proche de $100 \mathrm{~nm}$. La caractérisation (longueur de cohérence, distances interplanaires) a été faite par analyse de l'intensité et de la position des pics satellites en diffractométrie $\theta-2 \theta$. Les périodes ont été déterminées par réflectométrie $X$ aux petits angles. Tous les échantillons sont fortement texturés et la croissance s'effectue selon les plans réticulaires (111) pour le cuivre et le nickel et (110) pour le tungstène et le molybdène. Dans le cas des multicouches $\mathrm{Cu} / \mathrm{W}$, les épaisseurs $\mathrm{d}_{1}$ et $\mathrm{d}_{2}$ de chaque couche ont été choisies pour assurer une composition équiatomique. Le système $\mathrm{Cu} / \mathrm{W}$ n'étant pas miscible, il n'y a pas ou peu d'interdiffusion aux interfaces.

\section{RESULTATS ET DISCUSSION}

\subsection{Rappels théoriques}

Les vibrations longitudinales se propageant suivant l'axe d'empilement des couches dans un milieu multicouche périodique semi-infini obéissent à la relation de dispersion [4] :

$$
\cos (k d)=\cos \left(k_{1} d_{1}\right) \cos \left(k_{2} d_{2}\right)-\xi \sin \left(k_{1} d_{1}\right) \sin \left(k_{2} d_{2}\right)
$$

où $\mathrm{k}=\omega / \mathrm{V}$ et $\mathrm{d}=\mathrm{d}_{1}+\mathrm{d}_{2}$ sont respectivement le vecteur d'onde dans la super couche et la superpériode. $\xi=\frac{1}{2}\left(\frac{\rho_{1} V_{1}}{\rho_{2} V_{2}}+\frac{\rho_{2} V_{2}}{\rho_{1} V_{1}}\right)$ caractérise le contraste acoustique entre les deux couches. $\rho_{1}$ et $\rho_{2}$ sont les densités et $V_{1}$ et $V_{2}$, les vitesses du son des couches 1 et 2 respectivement. Les solutions réelles de l'équation (1) sont des modes de volume qui se propagent à l'intérieur de l'échantillon à une vitesse effective $V_{\text {eff }}$ dont l'expression est la limite de la relation (1) quand $\mathrm{kd} \rightarrow 0$, soit :

$$
V_{e n}=d\left[\frac{d_{1}^{2}}{V_{1}^{2}}+\frac{d_{2}^{2}}{V_{2}^{2}}+\left(\frac{Z_{1}}{Z_{2}}+\frac{Z_{2}}{Z_{1}}\right) \frac{d_{1} d_{2}}{V_{1} V_{2}}\right]^{-\frac{1}{2}}
$$


où $Z_{i}=\rho_{i} V_{i}$ représente l'impédance acoustique de la couche $i . V_{\text {eff }}$ est la pente de la courbe de dispersion au centre de la zone de Brillouin (Fig. 1).

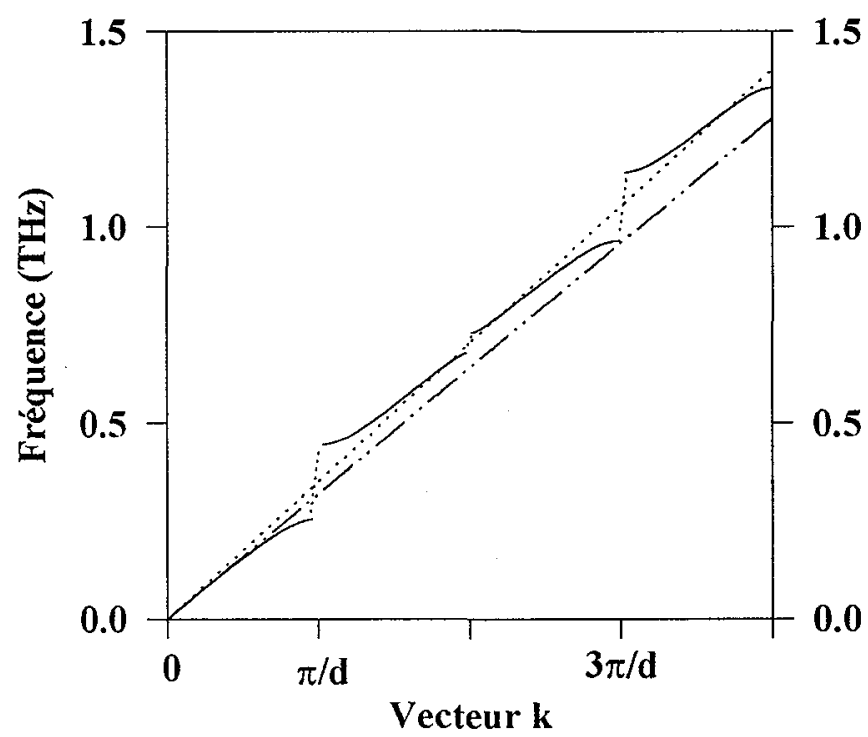

Figure 1 [4] : courbe de dispersion calculée pour Ies ondes acoustiques longitudinales dans un multicouche de $\mathrm{Cu} / \mathrm{W}$ dont l'épaisseur des couches est respectivement de $3,2 \mathrm{~nm}$ pour le cuivre et de $4,3 \mathrm{~nm}$ pour le tungstène. La courbe en pointillés est donnée par la moyenne harmonique des vitesses ultrasonores de $\mathrm{Cu}$ et de $\mathrm{W}$ et la courbe en tirets points par la moyenne harmonique des constantes élastiques.

Les solutions imaginaires de la relation (1) correspondent à des bandes de fréquences interdites à la propagation d'ondes ultrasonores. Ces gaps s'ouvrent à des fréquences définies par [5] :

$$
\begin{aligned}
& \operatorname{tg}\left(\frac{\omega_{(2 n+1)} d_{1}}{2 V_{2}}\right) \operatorname{tg}\left(\frac{\omega_{(2 n+1)} d_{2}}{2 V_{2}}\right)=\left(\frac{Z_{1}}{Z_{2}}\right)^{ \pm 1} \\
& \operatorname{tg}\left(\frac{\omega_{2 n} d_{1}}{2 V_{1}}\right)=-\left(\frac{Z_{1}}{Z_{2}}\right)^{ \pm 1} \operatorname{tg}\left(\frac{\omega_{2 n} d_{2}}{2 V_{2}}\right)
\end{aligned}
$$

pour les gaps de rang impair et pair respectivement. On montre [6] qu'à l'intérieur de chaque gap, il existe un mode de vibration localisé à la surface de l'échantillon et qui ne peut être excité que si le matériau constituant la surface libre est celui ayant la plus faible impédance acoustique (cuivre dans le cas des structures $\mathrm{Cu} / \mathrm{W}$ ). La fréquence de ces modes vérifie la relation :

$$
\operatorname{tg}\left(\frac{\omega_{100} d_{2}}{V_{2}}\right)+\frac{Z_{1}}{Z_{2}} \operatorname{tg}\left(\frac{\omega_{\text {loc }} d_{1}}{V_{1}}\right)=0
$$


Ainsi, la création d'une contrainte thermoélastique par absorption d'une impulsion laser ultra-brève à la surface d'une structure multicouche, donne naissance non seulement à des échos acoustiques qui se propagent à la vitesse du son effective mais aussi à des modes de vibration de très haute fréquence localisés près de la surface libre de l'échantillon.

\subsection{Résultats expérimentaux}

\subsubsection{Etude du changement de réflectivité aux temps longs : détermination des vitesses ultrasonores}

Nous présentons sur la figure 2 les changements de réflectivité en fonction du temps mesurés sur des multicouches $\mathrm{Cu} / \mathrm{W}$ de différentes périodes.

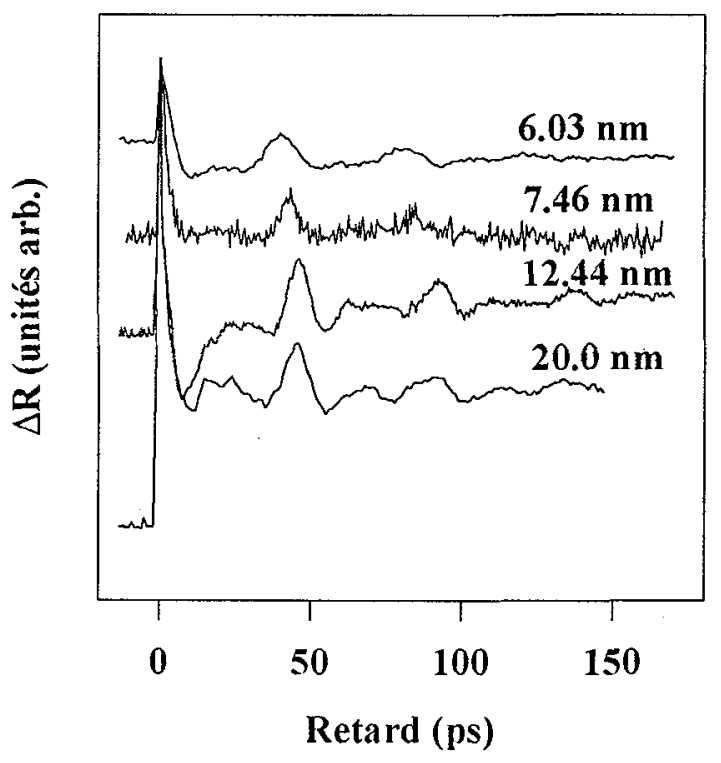

Figure 2 : courbes de réllectivité obtenus sur des multicouches de cuivre-tungstène [4]

Le pic principal à $\mathfrak{t}=0$ correspond à l'élévation instantanée de température provoquée par limpulsion pompe. Il est suivit par le refroidissement de la couche qui se traduit par une lente décroissance s'étendant sur plusieurs centaines de picosecondes. Des échos acoustiques se superposent à intervalles de temps réguliers sur ce fond continu. Seuls, les trois premiers échos sont clairement observés. La vitesse ultrasonore longitudinale est déduite du retard entre chaque échos. On a reporté sur la figure 3 (a) les variations de la vitesse du son en fonction de la superpériode pour la même série de multicouches de $\mathrm{Cu} / \mathrm{W}$.

Pour les périodes les plus grandes, les vitesses ultrasonores sont légèrement inférieures à la vitesse du son effective $(4726 \mathrm{~m} / \mathrm{s})$ déduite de la relation (2) en prenant pour $V_{1}$ et $V_{2}$ les vitesses du son dans le matériau massif correspondant et pour une direction de propagation selon l'axe cristallographique de croissance observé par diffraction $X$. L'accord est meilleur si l'on prend pour le cuivre une vitesse 
moyennée sur l'ensemble des directions cristallographiques. Cette correction est inutile pour le tungstène qui est presque isotrope du point de vue élastique. On attribue cette différence à la mosaïcité des couches. Pour les périodes les plus courtes, on observe un ramollissement des constantes élastiques. Ce phénomène est également observé dans d'autres systèmes métalliques [7]. On a également reporté sur la figure 3 (b) nos mesures effectuées sur des multicouches Ni/Mo. Ils présentent les mêmes effets. Cette anomalie est reliée à des effets interfaciaux [8].

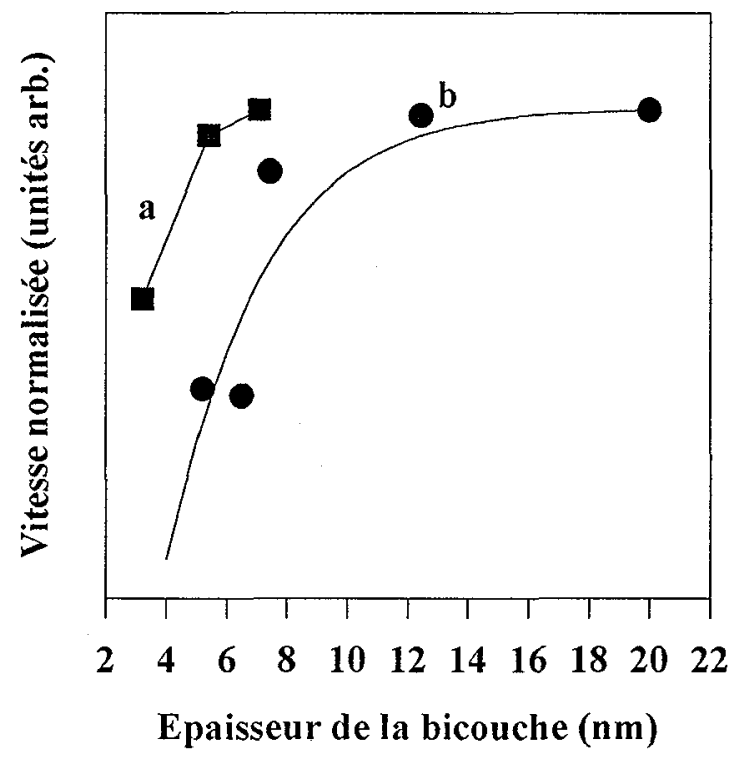

Figure 3 : vitesse ultrasonore dans des multicouclics de $\mathrm{Cu} / \mathrm{W}$ (a) et de Ni/Mo (b) en fonction de l'épaisseur d'une bicouche. Les deux lignes continues sont des aides visuclles.

\subsubsection{Etude du changement de réflectivité aux temps courts}

Outre les modes de volume dont il vient d'être question, certains échantillons présentent des modes de vibration de très haute fréquence ( $>500 \mathrm{GHz}$ ) qui se révèlent par une oscillation de la réflectance (Fig. 4).

Tableau 1 : fréquences des modes localisćs $\left(v_{l o c}\right)$ et des bords inférieurs de zone $(v)$ comparés aux résultats expérimentaux

\begin{tabular}{|c|c|c|c|c|}
\hline$d(n m)$ & $\begin{array}{c}\text { nombre de } \\
\text { bicouches }\end{array}$ & $v_{\text {exn }}(\mathrm{THz})$ & $v_{\text {loc }}(\mathrm{THz})$ & $v_{-}(\mathrm{THz})$ \\
\hline 6,03 & 13 & 0,810 & 0,828 & 0,811 \\
\hline 6,5 & 15 & 0,765 & 0,768 & 0,752 \\
\hline 7,46 & 12 & 0,662 & 0,669 & 0,656 \\
\hline 8,5 & 11 & 0,587 & 0,587 & 0,576 \\
\hline
\end{tabular}




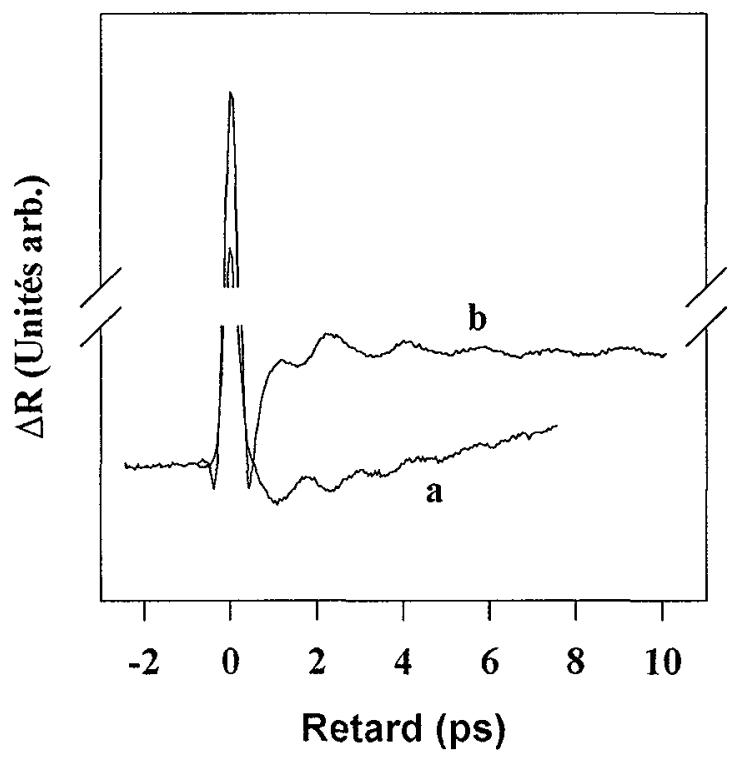

Figure 4 : Changement de réflectivité obtenu sur des temps courts pour un échantillon $\mathrm{Cu} / \mathrm{W}$ de période $6,03 \mathrm{~nm}$ (a) et sur un échantillon $\mathrm{Ag} / \mathrm{Ni}$ de période 7,5 $\mathrm{nm}$ (b). La fréquence de ces oscillations est déduite d'un ajustement effectué avec une sinusoide amortie. Elles est de $810 \mathrm{GHz}$ pour l'ćchantillon $\mathrm{Cu} / \mathrm{W}$ et de $583 \mathrm{GHz}$ pour $\mathrm{Ag} / \mathrm{Ni}$.

Ces oscillations prennent naissance immédiatement après l'absorption de l'impulsion pompe et s'amortissent sur quelques ps. Leur fréquence est inversement proportionnelle à la période de la multicouche. On a rassemblé dans le tableau 1 l'ensemble de nos résultats expérimentaux obtenus sur le système $\mathrm{Cu} / \mathrm{W}$, ainsi que les valeurs théoriques des modes localisés et de bord de zone du second gap déduites des relations (3) et (4). L'analyse de ce tableau montre que le mode excité pourrait être le mode localisé en surface.

\section{CONCLUSION}

Nous avons présenté les résultats obtenus sur une série de multicouches de différentes natures. Cette étude nous a permis de déterminer les vitesses ultrasonores dans ces structures et donc le module d'élasticité effectif du multicouche. Nous avons pu mettre en évidence un ramollissement de ce module lorsque l'épaisseur des couches diminue. Nous avons de plus détecté des modes de vibration acoustique confinés sous la surface. Le fort amortissement de ces modes pourrait apporter des informations sur la rugosité des interfaces.

\section{Remerciements.}

Les auteurs remercient K. F. Badawi, Ph. Goudeau, C. Jaouen et J. Pacaud (Univ. de Poitiers) pour leur avoir confié les différents échantillons. 


\section{Références}

[1] Carlotti G., Fioretto D., Socino G., Rodmacq B., and Pelosin V., J. Appl. Phys. 71, (1992) 4897-4902.

[2] Thomsen C., Grahn H. T., Maris H. J. and Tauc J., Phys. Rev. B34, (1986) 4129-4138.

[3] Goudeau Ph., Badawi K. F., Naudon A. and Gladyszewski G., Appl. Phys. Lett., 62, (1993) 246-248.

[4] Perrin B., Bonello B., Jeannet J. C. and Romatet E., Physica B 219 \& 220, (1996) 681-683.

[5] Grahn H. T., Maris H. J., Tauc J. and Abeles B., Phys. Rev. B38 (1988) 6066-6074.

[6] Chen W., Lu Y., Maris H. J. and Xiao G., Phys. Rev. B50 (1994) 14506-14515.

[7] Fullerton E. E., Schuller I. K., Parker F. T., Svinarich K. A., Eesley G. L., Bhadra R. and Grimsditch M., J. Appl. Phys. 73 (1993) 7370-7375.

[8] Clemens B. and Eesley G. L., Phys. Rev. Lett. 61 (1988) 2356-2359. 